\title{
El taller intergeneracional de huerto como propuesta de intervención para el alumnado con necesidades educativas especiales
}

\author{
The intergenerational workshop of orchard as a proposal for intervention \\ for students with special educational needs
}

\begin{abstract}
Resumen
En la actualidad, el alumnado con necesidades educativas especiales de los centros de educación especial requiere apoyos y medidas de alta intensidad e individualización para mejorar su desarrollo en las diferentes áreas de intervención y asegurar su máxima participación y vinculación con el entorno. Debido en parte a su historial de fracasos, los niños y niñas pueden presentar baja autoestima y autoconcepto, así como una limitación en las relaciones sociales, con tendencia al aislamiento. En este trabajo de investigación se plantea la realización de un taller intergeneracional de huerto entre el CEE APADIS y el Centro de Día de Alzheimer (Villena), como propuesta de intervención educativa para incrementar la autoestima, el autoconcepto, el bienestar emocional y las habilidades relacionales de los menores. Dentro de la investigación realizada se diferenciaron dos partes: evaluación pretest y postest a la realización del proyecto y valoración de las sesiones individuales del mismo. Los instrumentos empleados en la recogida de información fueron escalas fiables y validadas científicamente (algunas de ellas adaptadas) y también cuestionarios ad hoc. Una vez realizadas las sesiones de trabajo y analizados los resultados, se ha podido comprobar una mejora generalizada en todas las variables estudiadas, que demostraría la eficacia de los programas intergeneracionales como estrategia metodológica.
\end{abstract}

\section{Palabras clave}

Discapacidad intelectual, autoestima, habilidades relacionales, estado emocional, taller intergeneracional.

\begin{abstract}
Currently, students with special educational needs of special education centers require support and measures of high intensity and individualization to improve their development in the different areas of intervention and ensure their maximum participation and relationship with the environment. Due in part to their history of failures, children may have low self-esteem and self-concept, as well as a limitation in social relationships, with a tendency to isolation. In this research work, the realization of an Intergenerational Garden Workshop between the CEE APADIS and the Alzheimer's Day Center (Villena) is proposed, as an educational intervention proposal to increase self-esteem, self-concept, emotional well-being and skills Relational of minors. Research conducted within the two parties was formed: pretest and posttest assessment and project implementation and evaluation of individual sessions thereof. The instruments used in data collection were reliable and scientifically validated scales (some adapted) and ad hoc questionnaires. Once the work sessions have been carried out and the results analyzed, it has been possible to verify a general improvement in all the variables studied that demonstrates the effectiveness of intergenerational programs as a methodological strategy.
\end{abstract}

\section{Keywords}

Intellectual disability, self-esteem, relational skills, emotional state, intergenerational workshop.

\author{
Ignasi Navarro Soria \\ <ignasi.navarro@ua.es>
}

Universidad de Alicante. España

Virtudes Hernández Gandía <vhg.cee@apadis.com>

CEE APADIS (Villena). España

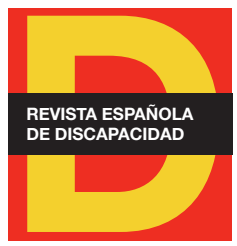

Para citar:

Navarro, I. y Hernández, V. (2020). "El taller intergeneracional de huerto como propuesta de intervención para el alumnado con necesidades educativas especiales". Revista Española de Discapacidad, 8(2), pp. 163-183.

Doi: <https://doi.org/10.5569/23405104.08.02.08>

Fecha de recepción: 11-10-2019 Fecha de aceptación: 31-08-2020

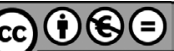




\section{Introducción}

La nueva conceptualización de la discapacidad intelectual se encuentra, según el DSM-5 (American Psychiatric Association (APA), 2013), dentro de los trastornos del neurodesarrollo. En ella se hace referencia a tres criterios básicos: déficit en el funcionamiento intelectual, déficit en el funcionamiento adaptativo y su inicio en los periodos iniciales del desarrollo. Desde este nuevo enfoque, la discapacidad intelectual se clasifica en base al funcionamiento adaptativo, y no en función del nivel de cociente intelectual como ocurría anteriormente.

En relación a la conducta adaptativa, conviene resaltar la importancia de los apoyos que pueden facilitarse al alumnado para mejorar la participación funcional, en cada uno de los entornos en los que se desenvuelva. Para ello, es fundamental la evaluación multidimensional dentro de su contexto y, a partir de ahí, determinar los sistemas de apoyo necesarios, así como su intensidad y duración (Luckasson et al., 2002).

En este sentido, el alumnado con pluridiscapacidad presenta unas necesidades de apoyo extenso o generalizado y requiere un enfoque multidisciplinar, dejando de lado las clasificaciones que atienden a una de las dimensiones de la persona como es el caso del cociente intelectual (Paternáin et al., 2009).

Se ha de tener en cuenta que cada persona con discapacidad intelectual tiene unas características singulares. No obstante, dificultades en las áreas de autonomía personal, de carácter cognitivo, de motricidadcorporal, del lenguaje y la comunicación, de tipo emocional o referidas a la interacción social; se encuentran presentes en todos los casos variando en función de la intensidad con la que precisan de un apoyo (Antequera et al., 2008).

La nueva concepción en el campo de la discapacidad intelectual está modificando no sólo la manera de ver a las personas con discapacidad intelectual, sino también la terminología empleada, las prácticas profesionales desarrolladas en este campo y las expectativas sobre los resultados de la persona (Schalock, 2009). La autodeterminación, entendida como la acción que capacita a cualquier persona para ser agente de su propia vida, está adquiriendo cada vez más protagonismo a nivel internacional tanto en la investigación como en la gestión de las organizaciones proveedoras de servicios. Este protagonismo también tiene su reflejo en España, ya que son muchas las instituciones que están intentando vertebrar sus entidades desde la aplicación transversal de conceptos como calidad de vida, autodeterminación y apoyos individualizados para mantener o mejorar la vida de las personas con discapacidad (Wehmeyer, 2005).

Resulta necesario que para el desarrollo de estas nuevas perspectivas en el ámbito escolar se tenga presente que además de las dificultades cognitivas, el alumnado con discapacidad intelectual leve o moderada presenta una autoestima y autoconcepto bajos acompañados de falta de motivación y creatividad. Además, sus relaciones sociales suelen ser limitadas y con tendencia al aislamiento, causado todo ello, por su historial de fracasos y la carencia de aceptación y refuerzo (Pérez-Cobacho y Tomás-Olivares, 2002).

Desde el punto de vista afectivo y emocional, es importante hacer hincapié en el hecho de que la autoestima, a pesar de que reúne un conjunto de características y actitudes de carácter interior de las personas, se forja habitualmente dentro del contexto de su vida social como la familia, la escuela, el trabajo, etc. (Pequeña y Escurra, 2006). 
En cuanto al autoconcepto, se trata de un conocimiento que no está presente en el momento del nacimiento, sino que es el resultado de un proceso activo de construcción por parte del sujeto a lo largo de todo su desarrollo evolutivo (Fernández y Goñi, 2008). Además, su desarrollo se produce de manera paralela y en interconexión con el desarrollo de otras facetas sociales o físicas (Amezcua y Pichardo, 2004).

Según lo anteriormente expuesto se puede entender que existen varios elementos fundamentales en el desarrollo del autoconcepto y la autoestima: la valoración recibida por otras personas, las experiencias vividas, las comparaciones sociales y, finalmente, la elaboración del marco interpretativo propiodon de toman especial relevancia las consecuencias originadas por las experiencias de éxito y de fracaso (Machargo, 1991).

La educación emocional es una innovación educativa que se justifica en las necesidades sociales y cuya finalidad es el desarrollo de competencias emocionales que contribuyan a un mejor bienestar personal y social (Bisquerra-Alzina, 2006). Por lo tanto, es un proceso educativo, continuo y permanente, y con una metodología eminentemente práctica que pretende potenciar el desarrollo de las competencias emocionales del alumnado con el objetivo final de capacitarlo para la vida y aumentar su bienestar personal y social (Bisquerra-Alzina y Pérez, 2007).

En este sentido, la educación emocional trata de mejorar la autoestima con expectativas realistas sobre sí mismo, desarrollar la capacidad de fluir y la capacidad para adoptar una actitud positiva ante la vida de manera que se posibilite un mayor bienestar subjetivo y que éste redunde en un mayor bienestar social (Fernández-Berrocal y Extremera, 2002).

Como recoge la Resolución de 18 de julio de 2018 del Diario Oficial de la Generalitat Valenciana, es una realidad que el proceso de inclusión educativa del alumnado con necesidades educativas especiales en los centros ordinarios del sistema educativo valenciano ha provocado cambios significativos en los centros de educación especial. Ha variado su perfil produciéndose la incorporación de alumnado con necesidades educativas especiales más graves que requiere apoyos especializados de alta intensidad e individualización durante toda la jornada escolar. Además, en la mayoría de los casos, la experiencia en el sistema educativo ordinario no ha sido del todo gratificante produciéndose situaciones de rechazo y de fracaso escolar. Por ello, los centros de educación especial se deben adaptar a esta realidad y plantear un marco más flexible y participativo que implique el desarrollo del proyecto educativo, del currículo y de la organización del centro, con el fin de facilitar la respuesta a la diversidad del alumnado, la implicación de los diferentes agentes y la vinculación del centro a su entorno.

Teniendo en cuenta todos los aspectos que se acaban de describir se propone la realización de un Taller Intergeneracional de Huerto con el objetivo de mejorar la autoestima, el autoconcepto, el bienestar emocional y las habilidades relacionales en este tipo de alumnado. En los centros educativos, el contacto con la naturaleza ofrece al alumnado experiencias directas de aprendizaje. A través de sus sentidos, de sus cuerpos, incorporan conocimientos que constituyen las bases de una memoria duradera, y son esenciales para su vitalidad. Al estar al aire libre entran en relación con otros organismos vivos y empiezan a conocerlos y respetarlos. Además, apreciar la importancia de su contribución al cuidado de animales y plantas, refuerza su identidad y les da autoconfianza (Freire, 2011).

Por otro lado, existen trabajos que han demostrado que los niños y niñas con un mayor contacto con la naturaleza afrontan mejor algunas de las situaciones adversas a las que suelen estar expuestos y que, además, sufren menos estrés (Corraliza y Collado, 2011). 
En esa misma línea, la investigación llevada a cabo por William Bird en numerosos centros educativos revela que salir al campo o a los parques y jardines regularmente mejora los resultados y la calidad de la enseñanza, ya que reduce los síntomas del déficit de atención, mejora los resultados académicos y el comportamiento de los alumnos y aumenta la motivación de los profesores (Freire, 2011).

El proyecto de huerto intergeneracional se plantea como un recurso social y didáctico que no solo facilita el aprendizaje de las tareas específicas del mismo y favorece un conocimiento socio-natural de su entorno, sino que en él se vincula a nuestros mayores, portadores del conocimiento que conforma nuestro patrimonio inmaterial, con los niños y niñas, como receptores directos de esa información (Bueno, 2004). Este hecho nos acerca al reto mundial de difundir, como parte del currículum en el marco de la educación formal, de algunos de los Objetivos de Desarrollo Sostenible de la Agenda 2030 establecidos por las Naciones Unidas derivados de problemáticas ecológicas, económicas y sociales (Dieste et al., 2019).

La participación de las personas mayores dentro del ámbito educativo formal viene justificada por los aspectos señalados en la legislación educativa. Así en la Ley Orgánica para la Mejora de la Calidad Educativa (LOMCE) se recoge la importancia de introducir nuevos patrones de conducta que ubiquen la educación en el centro de nuestra sociedad y economía, implicando a la sociedad civil en la transformación. Estas experiencias de intercambio intergeneracional promueven el empoderamiento del alumnado del centro y de los mayores reduciendo la fractura generacional.

Durante el año 2012, el Consejo de la Unión Europea declaró el Año Europeo del Envejecimiento Activo y la Solidaridad Intergeneracional. Con ello se pretende concienciar del rápido envejecimiento de la población, fomentar la convivencia entre generaciones y comprender que las personas mayores deben seguir ejerciendo un papel activo en la sociedad (Cordero del Castillo, 2012).

De esta manera, los programas intergeneracionales suponen la creación de espacios para el encuentro, la promoción del apoyo social y el intercambio de aprendizajes. También están encaminados a producir lazos afectivos entre las distintas generaciones que permiten a su vez la construcción de una sociedad más justa y solidaria (Sánchez et al., 2010).

La aplicación de este tipo de programas puede lograr numerosos beneficios a las personas participantes. Por un lado, en las personas mayores, se ha demostrado que se producen cambios en el humor, aumento de la vitalidad, mejora en el sentimiento de valía personal, huida del aislamiento o reconocimiento de su contribución a la comunidad por transmitir tradiciones y cultura. Por otra parte, en niños y niñas, se ha apreciado un incremento de la motivación, autoestima y confianza en uno mismo, menor soledad y aislamiento, así como un aumento del sentimiento de responsabilidad social (Pinazo y Kaplan, 2007).

Por último, cabe destacar que los programas intergeneracionales no sólo tienen un impacto positivo para las personas que participan directamente, sino que esta práctica es beneficiosa para todos los implicados en el programa: participantes, familia, profesionales y comunidad (Sánchez y Torrano, 2013).

Por todo esto, a través de este proyecto de investigación se comprobará si la puesta en marcha de un Taller Intergeneracional de Huerto con alumnado que presenta necesidades educativas especiales supone el cumplimiento los siguientes objetivos: 
1. Mejorar el autoconocimiento y autoconcepto aumentando la autoestima y el sentimiento de valía de los niños y niñas.

2. Incrementar la motivación produciendo una mejora en el estado emocional de los participantes.

3. Aumentar la cantidad y calidad de las interacciones sociales ayudando a desarrollar las habilidades relacionales.

\section{Método}

\subsection{Población}

Por tratarse este estudio, de una prueba piloto, la muestra es reducida y de tipo por conveniencia. La población escogida forma parte de las dos aulas de educación específica que se ofrece a colaborar en el estudio de Huerto Intergeneracional, sumando un total de 6 alumnos y alumnas del Centro de Educación Especial. Además, se incluyen 4 usuarios y usuarias del Centro de Día de Alzheimer, consiguiéndose un total de10 participantes.

En el proceso de selección de las personas que han formado parte del proyecto de investigación el requisito principal fue que, a pesar de tratarse de dos colectivos diferentes, presentaran características a nivel de desarrollo cognitivo, de personalidad y social, similares. Por lo tanto, se pudo componer un grupo homogéneo entre sí, con niveles semejantes de autonomía, comprensión y con intereses parecidos vinculados a la naturaleza. Atendiendo a la Global Deterioration Scale (GDS) (Reisberg, 1982) y como recogen Tárraga y Boada (2003), de los sujetos con demencia tipo alzhéimer, dos de ellos se encontraban en una fase de deterioro cognitivo muy leve o GDS 2, otro en fase de deterioro cognitivo leve o GDS 3 y el cuarto contaba con un deterioro cognitivo moderado o GDS 4. Además, dos de los usuarios del centro de día utilizaban bastón inglés como producto de apoyo para una movilidad más segura. El alumnado del colegio contaba con diagnóstico de plurideficiencia asociada a discapacidad intelectual y un nivel de competencia curricular entre los 5 a 7 años. Solo uno de ellos no tenía lenguaje oral, pero sí buena comprensión mediante pictogramas y gestos.

Respecto a la población escogida para la investigación, el Colegio de Educación Especial APADIS está formado por un total de 41 niños y niñas que acuden diariamente al centro, de los cuales se ha seleccionado un total de 6 alumnos y alumnas pertenecientes al aula verde y amarilla. Sus edades están comprendidas entre los 13 y los 19 años y, en general, presentan un importante desfase entre su edad mental y su edad cronológica.

En 4 de los casos, el padre y la madre están separados y en dos de ellos la familia está desestructurada, recibiendo supervisión y apoyo por parte de los equipos psicosociales de la Concejalía de Bienestar Social del municipio.

Las características esenciales que definen a este colectivo de personas son las siguientes:

- Nivel cognitivo: los procesos de aprendizaje son lentos y tienen una capacidad de atención reducida y con tendencia a la dispersión. Además, presentan déficit de memoria y una dificultad en el procesamiento de la información, en la resolución de problemas y en la toma de decisiones. 
- Nivel personal: muestran vulnerabilidad emocional e inseguridad, baja autoestima y autoconcepto pobre acompañado de falta de motivación y creatividad.

- Nivel social: en general se observa que sus relaciones sociales suelen ser limitadas y con tendencia al aislamiento causado, sobre todo, por la carencia de aceptación y refuerzo.

\subsection{Herramientas}

Atendiendo a las características tan especiales que presentaban los participantes del proyecto y de la muestra en particular, se produjo una búsqueda minuciosa de escalas y tests con rigurosa justificación científica pero que a la vez resultaran sencillas de completar y fáciles de interpretar.

A su vez también se elaboraron cuestionarios ad hoc para recoger información psicosocial sobre el estado emocional y la relación con otras personas en el contexto específico del huerto escolar.

Cabe resaltar que dentro de la investigación que se está describiendo, pueden diferenciarse dos partes: una de ellas corresponde a la evaluación pretest y postest de la realización del proyecto y otra a la valoración de las sesiones individuales del mismo. En la primera parte, tanto las escalas como el análisis de datos y resultados sólo se han aplicado a los niños y niñas con discapacidad intelectual como población específica escogida como muestra de nuestro proyecto y sobre la que están formulados los objetivos. Esta evaluación se llevó a cabo de forma individual durante la semana previa y posterior a la ejecución de las sesiones de trabajo del proyecto. En la segunda parte y de forma complementaria, el registro de valoración de cada sesión se pasó al total de los participantes para poder medir de forma inmediata y real si se producía algún cambio importante en el estado emocional, la motivación y las ganas de relacionarse con otras personas de cada sujeto (no se debe olvidar que debido a las características cognitivas de los participantes era de valiosa importancia recabar la información en el mismo momento de realizarse las diferentes actividades). Durante los primeros diez minutos de cada sesión, el alumnado y los mayores contaron con el apoyo verbal y visual (pictogramas) ofrecido por los profesionales para completar de manera autónoma los cuestionarios. Los cinco últimos minutos de cada sesión se dedicaron a rellenar el mismo cuestionario volviendo a valorar dichos ítems.

Las variables dependientes que se pretenden medir en esta investigación son la autoestima, el autoconcepto, la motivación, las habilidades relacionales y el estado emocional de los sujetos tomados como muestra. Todas las actividades han estado adaptadas a los participantes y en ellas se han utilizado diferentes estrategias o variables independientes encaminadas al desarrollo del bienestar emocional y al fortalecimiento de las relaciones sociales (la estrategia del reflejo, de la autoinspiración, el modelado con utilización del elogio y refuerzo positivo, los debates en grupo,...).

Los instrumentos empleados en la recogida de información han sido los siguientes:

1. Pretest y postest para la valoración de la repercusión del proyecto en la población escogida como muestra (los 6 niños y niñas con necesidades educativas especiales):

\section{- Escala de Autoestima de Rosenberg}

La primera escala utilizada para la investigación con el objeto de medir la autoestima en el alumnado con discapacidad intelectual ha sido la Escala de Autoestima de Rosenberg. 
Se trata de una de las escalas más utilizadas que incluye diez ítems cuyos contenidos se centran en los sentimientos de respeto y aceptación de sí mismo/a. La mitad de los ítems están enunciados positivamente y la otra mitad negativamente (Vázquez et al., 2004).

Esta prueba tiene fiabilidad para el conjunto de la muestra ya que el coeficiente alfa de Cronbach ofrece una puntuación de 0.87. De igual manera se describe su validez de constructo y para grupos conocidos.

Este test de autoestima tiene por objeto evaluar el sentimiento de satisfacción que la persona tiene de sí misma a través de 10 ítems, 5 de ellos enunciados de forma positiva y 5 de forma negativamente. Las opciones de respuesta están formuladas en una escala tipo Likert de 4 puntos, siendo A: Muy de acuerdo, B: De acuerdo, C: En desacuerdo y D: Muy en desacuerdo.

De los ítems 1 al 5, las respuestas A a D se puntúan de 4 a 1. De los ítems 6 al 10, las respuestas A a D se puntúan de manera inversa. Una vez realizado el sumatorio total, la puntuación oscila entre 10 y 40 , pudiéndose interpretar los resultados de la siguiente manera:

- De 30 a 40 puntos: Autoestima alta (considerada como autoestima normal).

- De 26 a 29 puntos: Autoestima media (no presenta problemas de autoestima graves, pero conviene mejorarla).

- Menos de 25 puntos: Autoestima baja (existen problemas significativos de autoestima).

\section{- Sección de Autoconocimiento de Escala de Autodeterminación INICO}

La siguiente prueba utilizada proporciona información precisa y válida sobre el nivel de autodeterminación de los estudiantes con discapacidad intelectual. Está formada por 61 ítems distribuidos en cuatro secciones que se corresponden con cada una de las dimensiones de autodeterminación propuestas por el Modelo Funcional de Wehmeyer (1999). Para la investigación se ha escogido únicamente la Sección de Autoconocimiento que consta de 10 ítems enunciados en primera persona y evalúan el conocimiento que el alumnado tiene de sus propias capacidades y limitaciones, así como la valoración personal o autoestima que tiene de sí mismo o misma.

El análisis de la consistencia interna de escala y las secciones que la forman se llevó a cabo mediante el índice alfa de Cronbach estandarizado obteniendo buenos resultados de fiabilidad (superior a .90) y con un Análisis Factorial Confirmatorio (AFC) que mostró un buen ajuste de los datos (Verdugo et al., 2015).

La aplicación de esta sección de la escala permite obtener datos sobre el conocimiento que el alumnado tiene de sus propias capacidades y limitaciones, así como la valoración personal o autoestima que tiene de sí mismo o misma para posteriormente, utilizar esta información en el diseño de planes de apoyo o intervención de la conducta autodeterminada.

Esta sección utiliza un formato de respuesta múltiple con cuatro opciones de respuesta basado en el grado de acuerdo (no estoy nada de acuerdo; no estoy de acuerdo; estoy de acuerdo; y estoy totalmente de acuerdo) y está acompañado de pictogramas para clarificar y facilitar el manejo de la escala. 


\section{- $\quad$ Adaptación de la Escala de Autoconcepto Forma 5}

El Autoconcepto Forma $5(A F-5)$ es un cuestionario de fácil aplicación que surge como una versión mejorada y actualizada de una forma precedente, el AFA (Autoconcepto Forma A). A la hora de diseñar el AF-5, se definieron 6 ítems de cada una de las 5 dimensiones (académica/ profesional, social, emocional, familiar y física) resultando un cuestionario total de 30 ítems con estructura pentadimensional. Tal y como citan los autores del cuestionario, éste se confeccionó sobre la base teórica del modelo multidimensional y jerárquico de la estructura del autoconcepto propuesta por los relevantes trabajos de Shavelson et al. (1976) y permite una completa medida de estos aspectos clave para el correcto desarrollo y bienestar de la persona (García y Musitu, 2001).

La dimensión de Autoconcepto académico hace referencia a la percepción que el individuo tiene de la calidad del desempeño de su rol como estudiante a partir de sus profesores y de sus cualidades. El Autoconcepto social ofrece información sobre la percepción que tiene el alumno o alumna de su desempeño en las relaciones sociales, por un lado, en lo que se refiere a su red social y por otro, a algunas cualidades de las relaciones interpersonales. En cuanto a la dimensión de Autoconcepto emocional, ésta alude a la apreciación del sujeto en cuanto al estado emocional y sus respuestas a situaciones específicas donde la persona implicada es de un rango superior. La cuarta dimensión es el Autoconcepto familiar y tiene relación con la percepción que tiene la persona de su implicación, participación e integración en el medio familiar. La última de ellas, el Autoconcepto físico, hace referencia a la percepción que tiene la persona de su aspecto y condición física.

En relación con la prueba utilizada en este proyecto y teniendo en cuenta la peculiaridad de la población de la muestra, se optó por la realización de una adaptación de la prueba que consistió en una simplificación de las opciones de respuesta con soporte visual de iconos de caras de colores para facilitar la elección por parte del alumnado. Así, los valores del 1 al 99 que había que asignar a cada ítem de la prueba original (desde muy en desacuerdo a muy de acuerdo) se transformaron en 5 opciones de respuesta tipo Likert simplificando su valor a $1,2.5,5,7.5$ y 10 .

En cuanto a la validez y fiabilidad de la prueba, en el manual de esta encontramos que, han sido múltiples las investigaciones que han respaldado la fiabilidad del AF-5 para ser considerada satisfactoria para validar otros instrumentos de autoestima y autoconcepto (Tomás y Oliver, 2004; Musitu y García, 2004; Núñez et al., 2007; Goñi y Ruiz, 2009). De manera específica, podemos decir que los resultados de la consistencia interna del cuestionario analizada mediante el coeficiente alfa de Cronbach han sido satisfactorios, ya que superan el considerado como valor de corte, con alfas que van desde el .71 de la escala autoconcepto social en la muestra femenina, al .89 del autoconcepto académico/laboral de la muestra masculina. La fiabilidad de la escala globalmente también ofrece resultados aceptables, en ambas muestras facilitando su aplicabilidad en diferentes contextos (Esnaola et al., 2011).

\section{- $\quad$ Adaptación de la Escala de Soledad UCLA revisada}

Dada la dificultad de producir experimentalmente grados diversos de soledad bajo condiciones controladas, resulta especialmente importante contar con instrumentos que permitan identificar personas con problemas de aislamiento y faciliten la cuantificación del aislamiento experimentado en la vida cotidiana, en contextos naturales. Autores como Russell et al. (1980) desarrollaron un instrumento para 
la evaluación de la soledad: la Escala de Aislamiento Social UCLA. Años más tarde, Vázquez y Jiménez (1994) adaptaron la escala a la población española de tercera edad en una versión que contaba con 20 ítems. Para el presente estudio, se decidió utilizar la adaptación de 10 preguntas de Russell (1996) y, además, tres escalas numéricas que miden: cantidad de contacto, sentimiento de soledad y satisfacción en las relaciones.

El cuestionario ha sido traducido directamente de su fuente original y consta de 10 preguntas para responder en línea, puntuables entre 1 y 4 puntos, en las que se ha de indicar la frecuencia en el grado de conformidad con los ítems propuestos. Ésta permite obtener una puntuación mínima de 10 y máxima de 40. Se considera que las puntuaciones más habituales oscilan entre 15 y 20, mientras que una puntuación superior a 30 sería indicativa de un grado severo de soledad.

Por otra parte, los valores asignados a las escalas numéricas complementarias a la prueba van desde el 1 (aspecto más negativo: aislamiento, soledad, insatisfacción) hasta el 10 (aspecto más positivo: numerosas relaciones sociales y satisfacción). Se consideran normales los valores comprendidos entre 20 y 25, mientras que valores por debajo de 15 pueden hacer sospechar un nivel de soledad o aislamiento social importante.

Además del uso de esta escala en relación con el estudio del fenotipo ampliado del autismo (Jobe y Williams, 2007), son interesantes los datos de la fiabilidad del coeficiente alfa de Cronbach donde se obtuvo un valor muy elevado $(0.95$, con un IC $95 \%$ de 0.94 a 0.98) demostrando también la validez de constructo con alta correlación entre ítems (Velarde-Mayol et al., 2016).

\section{- Cuestionario ad hoc sobre Huerto Intergeneracional}

Después de realizar una búsqueda infructuosa de escalas ya existentes que midieran el área concreta de participación en la actividad de huerto, se decidió elaborar un cuestionario ad hoc que intentara reflejar aquellos aspectos que más interesaban para analizar las respuestas de la población escogida como muestra.

Utilizando el mismo formato que en la adaptación de la Escala de Autoconcepto Forma 5 (cinco opciones de respuesta con soporte visual de iconos de caras de colores para facilitar la elección por parte del alumnado) se realizaron 23 ítems para recoger información sobre el nivel de satisfacción de participar en el proyecto, la percepción subjetiva de aprendizaje y aportación al grupo, y de las posibles repercusiones que este programa de Huerto Intergeneracional podía tener sobre ellos mismos y su entorno inmediato.

2. Registro antes de comenzar y al finalizar cada sesión de las siguientes variables: estado emocional, motivación y relación con otras personas.

Estos cuestionarios han sido cumplimentados personalmente por los 10 participantes con el apoyo de los profesionales presentes en casa sesión de trabajo. Se trata de una escala ad hoc, tipo Likert, donde las 5 opciones de respuesta corresponden a iconos de caras de colores con un código que va desde el grado 1 (rojo) muy enfadado/a, muy triste o nada hasta el grado 5 (verde oscuro) muy contento/a o mucho. 
Durante el transcurso de cada sesión de trabajo, se rellenó un registro diario por parte del docente donde por observación directa se recogió información sobre las actividades realizadas, las estrategias utilizadas, la respuesta conductual de los participantes y su nivel de socialización.

\subsection{Procedimiento}

En el proyecto de Huerto Intergeneracional han participado 6 niños y niñas del Colegio de Educación Especial APADIS junto a 4 usuarios y usuarias del Centro de Día de Alzheimer. Durante un mes, se han realizado ocho sesiones de trabajo. La primera de ellas sirvió para visitar el centro donde está ubicado el huerto escolar y para presentar el contenido del programa (en esta sesión no se recogieron registros de los participantes). Durante las siete sesiones restantes se respetó la misma secuencia de trabajo: recibimiento de los mayores, cumplimentación de registro de llegada, presentación y desarrollo de actividades, cumplimentación de registro de finalización y despedida.

Las tareas que se han realizado han tenido que ver con la puesta en marcha de las diferentes zonas del huerto (siembra y plantación / compostaje / invernadero) y otras diversas como la creación de un oasis de mariposas y la elaboración de aceite de caléndula. Todas las actividades han estado adaptadas a los participantes y en ellas se han utilizado diferentes estrategias encaminadas al desarrollo del bienestar emocional, de la autoestima y el autoconcepto y, además, el fortalecimiento de las relaciones sociales.

El proyecto ha contado con el respaldo de las direcciones de ambos centros y el consentimiento informado de las familias de los 10 participantes.

\section{Análisis de resultados}

Siguiendo la misma estructura utilizada en la descripción de los instrumentos que han servido para la recogida de la información, a continuación, se realizará la interpretación y análisis de los datos. En primer lugar, se describirán los resultados obtenidos en el pretest y postest de la repercusión que el proyecto ha tenido en la población escogida como muestra (los 6 niños y niñas con necesidades educativas especiales). Posteriormente, se analizarán los registros referentes al desarrollo de cada sesión cumplimentados por los 10 participantes (usuarios y usuarias del Centro de Día de Alzheimer y el alumnado del CEE APADIS).

Este primer bloque de escalas, se han pasado antes de empezar las sesiones de trabajo y una vez concluido el proyecto. El análisis de lo manifestado por la población de muestra llevará a interpretar más tarde si las variables dependientes escogidas de autoestima, autoconcepto y habilidades relacionales han sufrido cambios bajo la influencia de las variables independientes utilizadas como herramientas en el desarrollo del proyecto.

A continuación, en la Figura 1 se pueden observar los datos obtenidos en cuanto a la variable dependiente Autoestima en los 6 sujetos de la muestra. 
Figura 1. Nivel de autoestima

40

30

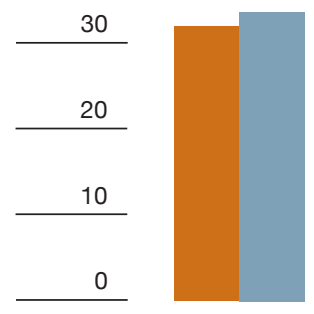

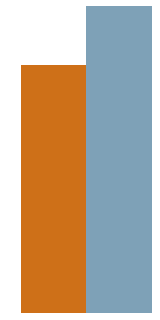

2

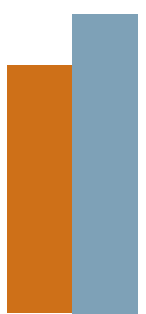

3

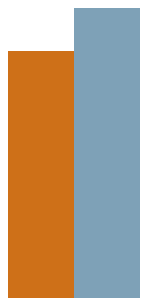

4

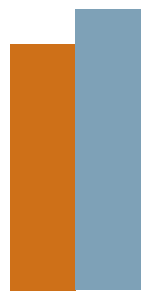

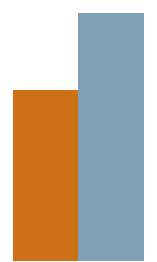

6
Antes del proyecto

Después del proyecto

Fuente: elaboración propia

El resultado de la aplicación del test de Rosenberg indica que en el $100 \%$ de los sujetos de la muestra, se ha producido un incremento del valor analizado y, por tanto, una mejora de su Autoestima una vez concluido el proyecto. Lo más significativo de estos resultados es que uno de los sujetos presentaba al inicio del programa un problema significativo de autoestima baja y 4 de ellos una autoestima media que convenía mejorar.

Durante el proceso de aplicación de la escala, se observó que los niños y niñas tenían dificultad para la comprensión del ítem número 1 "Siento que soy una persona digna de aprecio, al menos en igual medida que los demás". Por este motivo y para asegurar una comprensión correcta del significado del resto de ítems, se apoyó de forma oral la escala dando los sujetos incluso algunos ejemplos concretos sobre el significado de las cuestiones a las que debían responder.

En el caso de la población de muestra, tal y como puede verse en la Figura 2, de los 6 sujetos, tan sólo 1 de ellos presentaba un nivel de autoconocimiento dentro de la media antes de comenzar el proyecto, mientras que los otros 5 sujetos tenían un nivel bajo o muy bajo de autoconocimiento. Si comparamos los resultados una vez concluido el proyecto, puede observarse cómo 5 de los sujetos han mejorado esta variable de valoración personal o autoestima hasta niveles medios, encontrándose incluso 1 de ellos por encima de la media.

\section{Figura 2. Autoconocimiento}

$\begin{array}{r}6 \\ \hline 5 \\ \hline 4 \\ \hline 3 \\ \hline 2 \\ \hline 1 \\ \hline 0\end{array}$
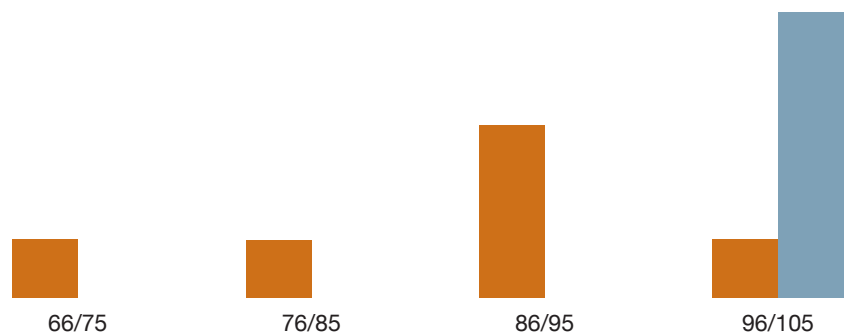

Antes del proyecto

Después del proyecto

Valores eje vertical: sujetos participantes; valores eje vertical: puntuación media normal 100

Fuente: elaboración propia. 
En el caso de la adaptación de la Escala de Autoconcepto Forma 5, interpretaremos los resultados obtenidos en términos de cambios significativos en las dimensiones medidas como puede verse en la Tabla 1.

\begin{tabular}{|c|c|c|c|c|c|c|c|c|c|c|}
\hline \multirow{2}{*}{ Participantes } & \multicolumn{2}{|c|}{ D. Académica } & \multicolumn{2}{|c|}{ D. Social } & \multicolumn{2}{|c|}{ D. Emocional } & \multicolumn{2}{|c|}{ D. Familiar } & \multicolumn{2}{|c|}{ D. Física } \\
\hline & Pre & Pos & Pre & Pos & Pre & Pos & Pre & Pos & Pre & Pos \\
\hline Sujeto 1 & 38.5 & 43.5 & 40 & 37.5 & 38.5 & 26 & 28.5 & 31 & 27.5 & 30 \\
\hline Sujeto 2 & 36 & 41 & 42.5 & 40 & 35 & 22.5 & 35 & 35 & 32.5 & 40 \\
\hline Sujeto 3 & 41 & 41 & 41 & 41 & 32.5 & 17.5 & 35 & 35 & 26 & 26 \\
\hline Sujeto 4 & 38.5 & 38.5 & 42.5 & 42.5 & 35 & 20 & 32.5 & 32.5 & 40 & 42.5 \\
\hline Sujeto 5 & 38.5 & 38.5 & 35 & 40 & 32.5 & 20 & 21.5 & 21.5 & 35 & 45 \\
\hline Sujeto 6 & 38.5 & 41 & 41 & 41 & 40 & 30 & 35 & 35 & 42.5 & 45 \\
\hline
\end{tabular}

Los valores del 1 al 99 del cuestionario original han sido simplificados a 5 opciones $(1,2.5,5,7.5,10)$.

Fuente: elaboración propia.

En relación con los resultados obtenidos en esta investigación, la dimensión de Autoconcepto académico se mantiene estable en todos los sujetos sin presentar cambios al comparar el pretest y postest.

En el apartado de Autoconcepto social, los resultados derivados de la investigación sugieren mayor disparidad en las respuestas no pudiéndose establecer una pauta común en los sujetos.

En cuanto a la dimensión de Autoconcepto emocional, de los resultados alcanzados se desprende que en todos los participantes ha habido una disminución en la puntuación una vez terminado el proyecto. Esto indica un autoconcepto emocional mayor, como respuesta a una mejora en el control de las situaciones planteadas a lo largo del proyecto y una respuesta adecuada a la ansiedad y el nerviosismo en las diferentes sesiones de trabajo.

En el apartado de Autoconcepto familiar y, puesto que el desarrollo del proyecto no tiene que ver directamente con el ámbito familiar, los resultados obtenidos se mantienen prácticamente invariables en el pretest y el postest.

Las respuestas obtenidas en el postest con relación a la última dimensión de la escala de Autoconcepto físico indican una mejora en las puntuaciones de todos los sujetos relacionadas con la percepción física que tienen de sí mismos según la opinión de los demás.

Del análisis de los datos de la Adaptación de la Escala de Soledad UCLA revisada se concluye, como puede verse en la Tabla 2, que tan sólo uno de los sujetos presentaba antes del proyecto un grado de soledad elevado que mejora tras el proyecto. En el resto de los sujetos, no existen diferencias significativas en el pretest y postest. 


\begin{tabular}{l}
\hline Tabla 2. Escala Soledad UCLA Revisada (Adaptación) \\
\begin{tabular}{|l|c|c|}
\hline \multirow{2}{*}{ Participantes } & \multicolumn{2}{c|}{ Puntuación obtenida } \\
\cline { 2 - 3 } & Pretest & Postest \\
\hline Sujeto 1 & 19 & 18 \\
\hline Sujeto 2 & 21 & 20 \\
\hline Sujeto 3 & 19 & 19 \\
\hline Sujeto 4 & 20 & 18 \\
\hline Sujeto 5 & 26 & 21 \\
\hline Sujeto 6 & 20 & 20 \\
\hline
\end{tabular}
\end{tabular}

Valores obtenidos de cuestionario en línea en la página web http://espectroautista.info/ (2016).

Fuente: elaboración propia.

En cuanto a las tres escalas numéricas complementarias, como puede verse en la Tabla 3, en el total de los sujetos se aprecia una mejora en los ítems planteados, siendo especialmente significativa en el mismo sujeto que presentaba un grado de soledad elevada antes de la realización del proyecto.

Tabla 3. Escala Soledad UCLA Revisada (Adaptación). Escalas Numéricas

\begin{tabular}{|l|c|c|}
\hline \multirow{2}{*}{ Participantes } & \multicolumn{2}{|c|}{ Puntuación obtenida } \\
\cline { 2 - 3 } & Pretest & Postest \\
\hline Sujeto 1 & 22 & 27 \\
\hline Sujeto 2 & 26 & 27 \\
\hline Sujeto 3 & 23 & 26 \\
\hline Sujeto 4 & 23 & 24 \\
\hline Sujeto 5 & 11 & 21 \\
\hline Sujeto 6 & 24 & 25 \\
\hline
\end{tabular}

Valores globales obtenidos tras la suma de los tres ítems planteados (cantidad de contacto, sentimiento de soledad y satisfacción en las relaciones).

Fuente: elaboración propia.

Por lo que respecta al Cuestionario ad hoc sobre Huerto Intergeneracional se han obtenido resultados muy similares en la totalidad de los sujetos, sin importar el género, ni que dos de ellos jamás hubieran participado en la actividad escolar de huerto.

El $100 \%$ del alumnado expresó estar totalmente de acuerdo por participar en la actividad intergeneracional mientras que en el pretest un 33,3\% del grupo se mostraban indecisos. Se ha comprobado en el postest que el 83,3\% estaba de acuerdo en haber aportado algo a los demás participantes, mientras que el $100 \%$ expresó su total conformidad con haber aprendido algo del resto. Además, en cuanto al hecho de si participar en tareas desarrolladas en el entorno natural y con personas de mayor edad, les había proporcionado 
bienestar y gran motivación, en el postest el 83,3\% se mostró totalmente de acuerdo y el 16,6 \% de acuerdo, a pesar de que los datos registrados previamente mostraban que el $50 \%$ del total se mostró indeciso en estas cuestiones.

Por último, contando con una cifra en el pretest del 33,3\% de indecisos sobre si les parecía suficiente el número de sesiones de trabajo, el postest arrojó un resultado del 83,3\% totalmente en desacuerdo y el $16,6 \%$ en desacuerdo con la cantidad de sesiones, ya que les hubiese gustado disfrutar de más sesiones de huerto y hubiesen deseado poder continuar la actividad durante todo el curso escolar. El $100 \%$ de los niños y niñas mejoraron su grado de satisfacción al considerar que la participación en este taller les reportó beneficios y sirvió para establecer lazos entre generaciones.

Se realizará a continuación el análisis del segundo bloque de cuestionarios que tienen que ver con el desarrollo de las 7 sesiones y fueron cumplimentados tanto al inicio como al final de cada día por los 10 participantes. Como se ha nombrado anteriormente, los resultados obtenidos de estas escalas ad hoc a pesar de no estar validadas, son muy significativos ya que muestran de forma inmediata y veraz la evolución de cada variable (estado anímico, motivación y relación con otras personas) sesión tras sesión, información que en el caso de personas con las características de los dos colectivos que participan es imprescindible valorar.

Como puede verse en la Figura 3, el estado emocional del $100 \%$ de los participantes mejoró en todas las sesiones. Si bien en alguna sesión alguno de los participantes manifestó malestar al inicio, al finalizar la actividad, esta situación se había revertido.

\section{Figura 3. Estado emocional}

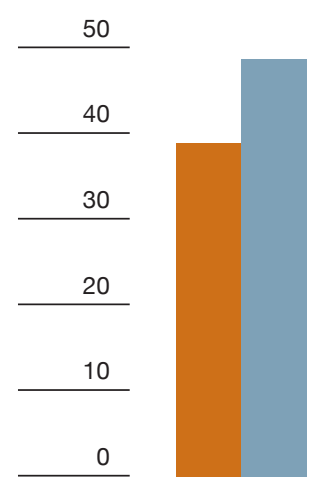

S1

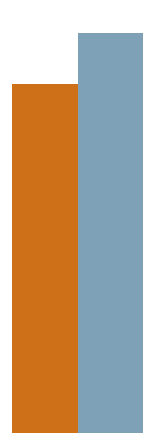

S2

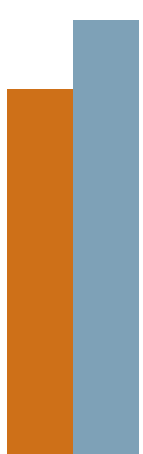

S3

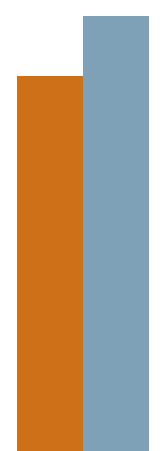

S4

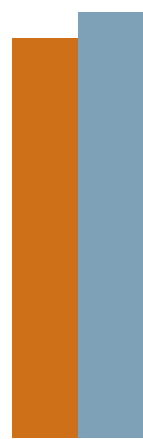

S5

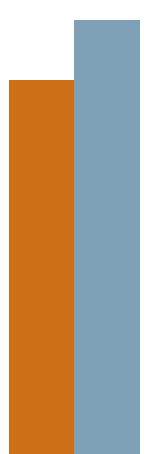

S6

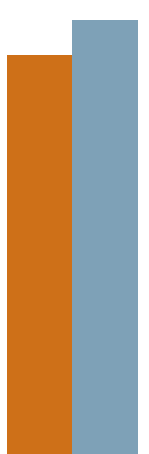

S7

Valores del eje vertical: 0-10 Muy triste, 11-20 Triste, 21-30 Indiferente, 31-40 Contento/a, 41-50 Muy contento/a.

Fuente: elaboración propia.

Tal como se observa en la Figura 4, se obtiene el mismo resultado con la variable de motivación. En la totalidad de los casos se puede apreciar un aumento considerable de la motivación al final de cada sesión y, además, un cambio importante al comparar el inicio de la sesión 1 y la 7. 


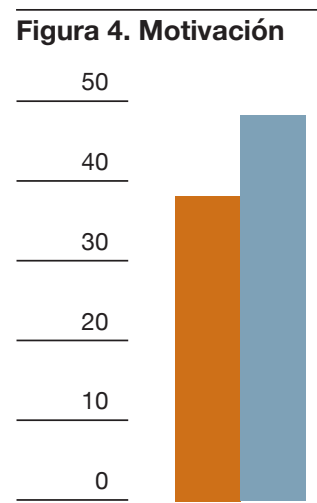

S1

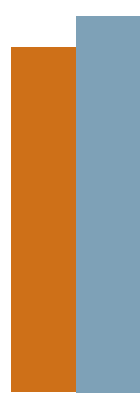

S2

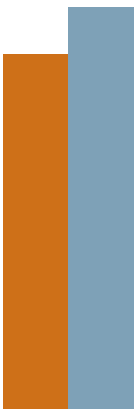

S3

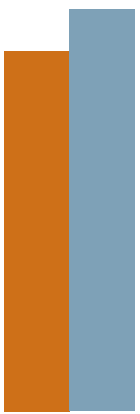

S4

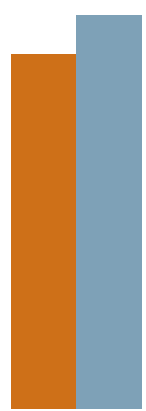

S5

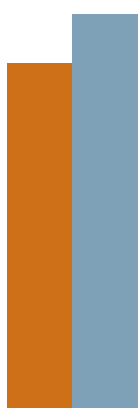

S6

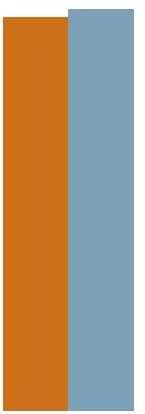

S7

Valores del eje vertical: 0-10 Ninguna, 11-20 Poca, 21-30 Normal, 31-40 Algo, 41-50 Mucha.

Fuente: elaboración propia.

En cuanto a las ganas que los participantes tienen para relacionarse con otras personas, la mejora en la predisposición al inicio de la sesión respecto al término de la misma vuelve a incrementar su valor produciéndose una mejora de la variable (véase la Figura 5).

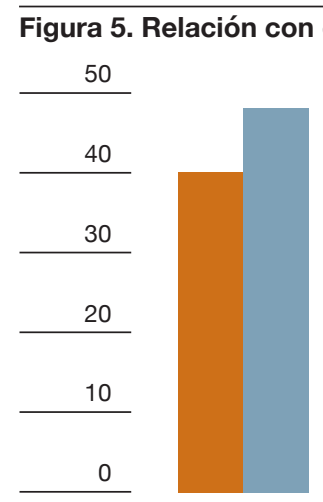

S1

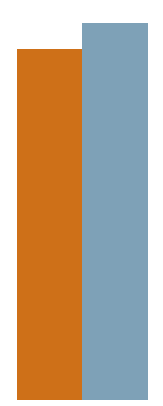

S2

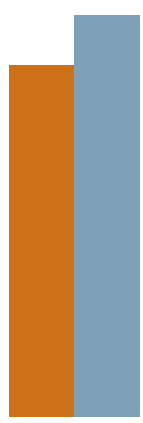

S3

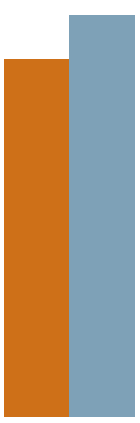

S4

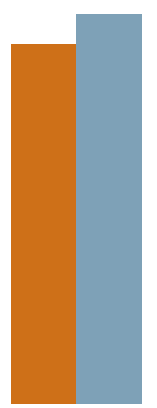

S5

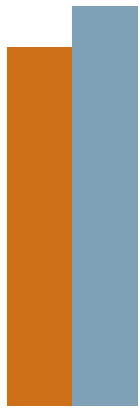

S6

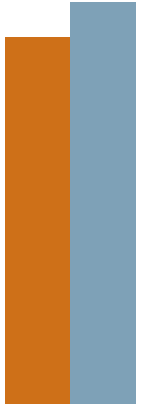

S7
Inicio sesión

- Final sesión

Valores del eje vertical: 0-10 Nada, 11-20 Poco, 21-30 Normal, 31-40 Algo, 41-50 Mucho.

Fuente: elaboración propia.

De la información recogida por observación directa a través del registro diario del docente se pueden extraer aspectos tan significativos como la iniciativa por salir a recibir a la puerta a los usuarios del Centro de Alzheimer, muestras constantes de cariño entre los participantes (dar la mano, acompañar cogiendo del brazo, saludos, sonrisas, besos, abrazos,...), interesarse por la falta de asistencia o el retraso de algún compañero o compañera, verbalizar emociones como "estoy nerviosa como cuando vienen los Reyes Magos", "cada vez que vengo al huerto se me abre el corazón", "me hubiera pesado no venir", "mejor nos sentamos mezclados ¿no?”... 


\section{Discusión}

En cuanto al primer objetivo planteado sobre el aumento de la autoestima y el sentimiento de valía a partir de la mejora del autoconcepto, se puede confirmar que en este caso se cumple ya que, en los resultados obtenidos en el postest de las escalas, ambas variables han incrementado de forma notable sus valores.

Tal y como señalaban Pequeña y Escurra (2006), este conjunto de características y actitudes se forja dentro del contexto escolar y social del niño o la niña, siendo determinantes en esta mejora las relaciones que han tenido durante el programa con los demás participantes y su entorno.

Atendiendo a lo descrito por Fernández y Goñi (2008) el alumnado ha mejorado la percepción sobre sí mismo gracias a los refuerzos positivos y elogios que han recibido por parte de los mayores y los profesionales implicados, produciéndose un proceso activo en la construcción de su imagen que ha ayudado notablemente a la manifestación de sus deseos e inquietudes.

En ese sentido, los niños y niñas con necesidades educativas especiales han sido capaces de describir de manera positiva algunas de sus habilidades y talentos con relación a las actividades llevadas a cabo en el huerto intergeneracional, como muestra del desarrollo del autoconcepto de manera paralela y en interconexión con la faceta social a la que apuntaban Amezcua y Pichardo (2004). Por tanto, se coincide con Machargo (1991) en que la realización exitosa de esta experiencia de huerto ha generado en el alumnado un sentimiento de eficacia y valía que les ha llevado a verse de forma positiva, a confiar en sus propias capacidades para solucionar problemas sencillos y a comenzar a proponerse metas alejadas del fracaso al que tantas veces se han visto abocados.

Con relación al segundo objetivo sobre la mejora de la motivación y el estado emocional de los participantes, se puede afirmar que vuelve a cumplirse quedando demostrado con el resultado de los diferentes instrumentos del programa. De esta forma y partiendo de las características descritas por Pérez-Cobacho y Tomás-Olivares (2002) en cuanto a la falta de motivación y creatividad que acompaña a las personas con discapacidad intelectual a causa de una baja autoestima, además de la necesidad del desarrollo en el ámbito escolar de las diferentes competencias emocionales (Bisquerra-Alzina y Pérez, 2007), el resultado obtenido tanto en el postest del registro diario cumplimentado por los participantes como en el cuestionario ad hoc del Taller Intergeneracional de Huerto, muestra que dichas variables aumentaron en el total de los participantes del programa. Como recogen las notas del registro de observación diaria del docente e indicaba Bueno (2004) ha sido muy significativa la alegría, el respeto y el cariño que constantemente se han demostrado niños y mayores en cada sesión de trabajo.

En referencia a lo aportado por Wehmeyer (2005), se ha observado también un mayor grado de autodeterminación en los niños y niñas como sujetos capaces de actuar y mostrar una gran predisposición a la hora de desarrollar tareas novedosas. A esto ha ayudado el aumento en la seguridad para la realización de las diversas actividades en el ámbito natural (Freire, 2011), el hecho de despertar nuevos intereses para ir ampliando sus conocimientos y experiencias y el autorrefuerzo.

Los resultados obtenidos en la investigación confirman que el tercer objetivo planteado se cumple de igual manera que los anteriores. Si bien es cierto que la Escala de Soledad UCLA revisada no ha ofrecido cambios 
significativos en el pretest y postest, del resto de datos registrados sí puede desprenderse una mejora en las habilidades relacionales del alumnado y, especialmente, en las ganas de participar en el taller afrontando las situaciones novedosas con menos estrés como apuntaban Corraliza y Collado (2011).

El importante desarrollo de la competencia social al que hacían referencia Bisquerra y Pérez (2007), junto con los beneficios de los programas intergeneracionales descritos por Pinazo y Kaplan (2007), quedan sobradamente reflejados en aspectos tan importantes como la voluntad de ayudarse mutuamente, de declinar la participación en otras actividades de centro por dar prioridad al taller intergeneracional y en sentirse partícipes de acciones de mejora del medio ambiente que suponen en reconocimiento del resto de compañeros del centro.

Tal y como mencionaban Sánchez et al. (2010), el hecho de querer asistir cada día a la sesión de trabajo y hacérselo saber a profesionales y familiares demuestra, sin lugar a duda, la creación de lazos afectivos y beneficios mutuos como camino hacia la construcción de una sociedad más justa, integrada y solidaria.

\section{Limitaciones de la investigación}

Conviene mencionar que, debido al tamaño reducido de la muestra y la especificidad de las características de los sujetos, cabe considerar este proyecto como un estudio de caso, sin resultar recomendable extrapolar los resultados aportados a otros contextos generales.

Por otro lado, se ha encontrado dificultad en la búsqueda y elección de escalas específicas para este colectivo que resultaran sencillas de rellenar por los niños y niñas y que además fueran fáciles de interpretar. Por este motivo se han tenido que adaptar algunas de ellas y realizar cuestionarios ad hoc, lo que ha supuesto un esfuerzo añadido. Antes de comenzar el proyecto, se temió por la acogida entre los participantes (tenía carácter voluntario y además era necesario el consentimiento de familiares), por los posibles problemas de salud de los mayores que pudieran causar baja y porque las inclemencias del tiempo forzaran a realizarlo a cubierto perdiendo la oportunidad de estar al aire libre. Por suerte para todos y todas, esta preocupación inicial pudo resolverse de forma satisfactoria.

\section{Aplicación práctica futura}

Como resultado de la gran aceptación manifestada por los participantes y de su voluntad expresa de seguir asistiendo al taller, sería recomendable considerar para un futuro próximo la continuidad del proyecto durante todo el curso escolar por lo menos una vez a la semana. De esta forma, podría beneficiarse un mayor número de participantes de ambos colectivos, así como incorporar la participación directa de sus familiares. 
En cuanto al proceso de investigación, esta consolidación del Taller Intergeneracional de Huerto permitiría aumentar el tamaño de la muestra para lograr extraer conclusiones generalizables, profundizar en las variables estudiadas hasta el momento e incorporar otras nuevas. 
American Psychiatric Association (APA) (2013). Diagnostic and statistical manual of mental disorders, fifth edition (DSM-5). Arlington, VA (USA): American Psychiatric Publishing.

Amezcua, J. A. y Pichardo, L. (2004). “Autoconcepto y construcción personal en la educación personal”. En M. V. Trianes y J. A. Gallardo (coords.), Psicología de la Educación y del Desarrollo en Contextos Escolares (pp. 181-192). Madrid: Pirámide.

Antequera, M. et al. (2008). Manual de atención al alumnado con necesidades específicas de apoyo educativo derivadas de discapacidad intelectual. Sevilla: Junta de Andalucía. Recuperado de http://hdl.handle. net/11162/3180.

Bisquerra-Alzina, R. (2006). "Orientación psicopedagógica y educación emocional”. Estudios Sobre Educación, 11, pp. 9-25. Recuperado de http://hdl.handle.net/10171/9208.

Bisquerra-Alzina, R. y Pérez, N. (2007). “Las competencias emocionales”. Educación XXI, 10, pp. 61-82. DOI: http://doi.org/10.5944/educxx1.1.10.297.

Bueno, M. (2004). El huerto familiar ecológico. La gran guía práctica del cultivo natural. Barcelona: RBA Editores.

Comunidad Valenciana. Resolución de 18 de julio de 2018, del secretario autonómico de Educación e Investigación, por la que se dictan instrucciones para la organización y funcionamiento de los centros de Educación Especial sostenidos con fondos públicos para el curso 2018-2019. Diario Oficial de la Generalitat Valenciana, de 20 de julio de 2018, núm. 8343, pp. 30987-30997. Recuperado de https://www.dogv.gva.es/datos/2018/07/20/ pdf/2018_7182.pdf.

Cordero del Castillo, P. (2012). "2012, Año Europeo del Envejecimiento Activo y la Solidaridad Intergeneracional”. Humanismo y trabajo social, 11, pp.101-117.

Corraliza, J. A. y Collado, S. (2011). "La naturaleza cercana como moderadora del estrés infantil". Psicothema, 23(2), pp. 221-226. Recuperado de http://www.unioviedo.es/reunido/index.php/PST/article/view/9026/8890.

Dieste, B., et al. (2019). "Inclusión de los Objetivos de Desarrollo Sostenible en el Currículum de Educación Primaria y Secundaria en Escuelas Rurales de Zaragoza”. Revista Internacional de Educación para la Justicia Social (RIEJS), 8(1), pp. 97-115. DOI: http://doi.org/10.15366/riejs2019.8.1.006.

Esnaola, I. et al. (2011). "Propiedades psicométricas del cuestionario de Autoconcepto AF5". Anales de Psicología, 27(1), pp. 109-117. Recuperado de https://revistas.um.es/analesps/article/view/113531.

España. Ley Orgánica 8/2013, de 9 de diciembre, para la Mejora de la Calidad Educativa (LOMCE). Boletín Oficial del Estado, 10 de diciembre de 2013, núm. 295, pp. 97858-97921. Recuperado de https://www.boe.es/boe/ dias/2013/12/10/pdfs/BOE-A-2013-12886.pdf.

Espectro Autista Info (2016). La Escala de Soledad de UCLA (ULS). Recuperado de http://espectroautista.info/ ULS-es.html.

Fernández, A. y Goñi, E. (2008). "El autoconcepto infantil: una revisión necesaria". International Journal of Developmental and Educational Psychology, 2 (1), pp. 13-22. Recuperado de http://www.redalyc.org/articulo. oa? id=349832317001. 
Fernández-Berrocal, P. y Extremera, N. (2002). "La inteligencia emocional como una habilidad esencial en la escuela". Revista Iberoamericana de Educación, 29(1), pp. 1-6. DOI: http://doi.org/10.35362/rie2912869.

Freire, H. (2011). Educar en verde: Ideas para acercar a niños y niñas a la naturaleza (Vol. 21). Barcelona: Graó.

García, F. y Musitu, G. (2001). Autoconcepto Forma 5. AF5. Manual. Madrid: TEA.

Goñi, A. y Ruiz de Azúa, S. (2009). "La estructura interna del autoconcepto físico”. En A. Goñi (coord.), El autoconcepto físico: psicología y educación (pp. 81-97). Madrid: Pirámide.

Jobe, L. E. y Williams White, S. (2007). "Loneliness, social relationships, and a broader autism phenotype in college students". Personality and Individual Differences, 42(8), pp. 1479-1489. DOI: http://doi.org/10.1016/j. paid.2006.10.021.

Luckasson, R. et al. (2002). Mental Retardation. Definition, classification and systems of supports. $10^{\mathrm{a}}$ ed. Washington, DC: AAMR.

Machargo, J. (1991). El profesor y el autoconcepto de sus alumnos. Teoría y práctica. Madrid: Escuela Española.

Musitu, G. y García, J. F. (2004). "Consecuencias de la socialización familiar en la cultura española”. Psicothema, 16(2), pp. 288-293. Recuperado de http://www.redalyc.org/articulo.oa?id=72716218.

Núñez, J. L. et al. (2007). "Análisis de las propiedades psicométricas del cuestionario Autoconcepto Forma 5 en estudiantes universitarios”. Estudios de Psicología, 28(3), pp. 333-342. DOI: http://doi.org/10.1174/02109390 7782506461.

Paternáin, C. et al. (2009). Alumnado con pluridiscapacidad: orientaciones para la estructuración espacio temporal en aulas alternativas. Navarra: Gobierno de Navarra.

Pequeña, J. y Escurra, L. M. (2006). "Efectos de un programa para el mejoramiento de la autoestima en niños de 8 a 11 años con problemas específicos de aprendizaje”. Revista II PSI Facultad de Psicología, 9(1), pp. 9-22.

Pérez-Cobacho, J. y Tomás-Olivares, V. (2002). "Aspectos evolutivos del niño con discapacidad intelectual". En J. M. García-Fernández et al. (coords.), Discapacidad intelectual: desarrollo, comunicación e intervención (pp. 51-67). Madrid: Editorial CEPE.

Pinazo, S. y Kaplan, M. (2007). "Los beneficios de los programas intergeneracionales”. En M. Sánchez (dir.), Programas intergeneracionales. Hacia una sociedad para todas las edades (pp. 70-101). Barcelona: Fundación La Caixa.

Reisberg, B. et al. (1982). "The Global Deterioration Scale for assessment of primary degenerative dementia". The American Journal of Psychiatry,139(9), pp. 1136-1139.

Russell, D. et al. (1980). "The Revised UCLA Loneliness Scale: Concurrent and Discriminant Validity Evidence". Journal Personality Social and Psychology, 39, pp. 472-480.

Russell, D. W. (1996). "UCLA Loneliness Scale (Version 3): Reliability, Validity, and Factor Structure". Journal of Personality Assessment, 66(1), pp. 20-40. DOI: http://doi.org/10.1207/s15327752jpa6601_2.

Sánchez, M. et al. (2010). Programas intergeneracionales. Guía introductoria. Madrid: IMSERSO.

Sánchez, M. G. y Torrano, D. H. (2013). "Los beneficios de los programas intergeneracionales desde la perspectiva de los profesionales". Pedagogía Social: Revista Interuniversitaria, 21, pp. 213-235. Recuperado de http:// www.redalyc.org/articulo.oa?id=135029517010. 
Schalock, R. L. (2009). "La nueva definición de discapacidad intelectual, apoyos individuales y resultados personales”. Revista Española sobre Discapacidad Intelectual FEAPS Siglo Cero, 40(1), pp. 22-39. Recuperado de http://hdl.handle.net/11181/3841.

Shavelson, R. J. et al. (1976). "Self-Concept: Validation of Construct Interpretations". Review of Educational Research, 46, pp. 407-441. DOI: http://doi.org/10.3102/00346543046003407.

Tàrraga, L., y Boada, M. (2003). Ejercicios prácticos de estimulación cognitiva para enfermos de Alzheimer en fase moderada. Barcelona: Glosa.

Tomás, J. M. y Oliver, A. (2004). "Confirmatory factor analysis of a Spanish multidimensional scale of self-concept”. Revista Interamericana de Psicología, 38, pp. 285-293. Recuperado de http://www.redalyc.org/articulo. oa?id=28438214.

Vázquez, A. J. et al. (2004). “Escala de autoestima de Rosenberg: fiabilidad y validez en población clínica española”. Apuntes de Psicología, 22(2), pp. 247-255.

Vázquez, A. J. y Jiménez, R. (1994). "Rules: Escala de soledad UCLA revisada. Fiabilidad y validez de una versión española". Revista de Psicología de la Salud, 6(1), pp. 45-54.

Velarde-Mayol, C. et al. (2016). "Validación de la escala de soledad de UCLA y perfil social en la población anciana que vive sola". SEMERGEN-Medicina de Familia, 42(3), pp. 177-183. DOI: http://doi.org/10.1016/j.semerg.2015.05.017.

Verdugo, M. A. et al. (2015). Escala ARC-INICO de Evaluación de la Autodeterminación. Manual de Aplicación y Corrección. Salamanca: Instituto Universitario de Integración en la Comunidad.

Wehmeyer, M. L. (1999). "A Functional Model of Self-determination: Describing Development and Implementing Instruction". Focuson Autism and Other Developmental Disabilities, 14, pp. 53-62. DOI: http://doi.org/10.1177 /108835769901400107.

Wehmeyer, M. L. (2005). "Self-determination and individuals with severe disabilities: Re-examining meanings and misinterpretations". Research and Practice for Persons with Severe Disabilities, 30(3), pp.113-120. DOI: http:// doi.org/10.2511/rpsd.30.3.113. 